\title{
AORTIC HOMOGRAFT INTERPOSITION FOR MANAGEMENT OF COMPLETE TRACHEAL ANASTOMOTIC DISRUPTION AFTER HEART-LUNG TRANSPLANTATION
}

\author{
Timothy M. Hoffman, MD, ${ }^{\mathrm{a}}$ J. William Gaynor, MD, ${ }^{\mathrm{b}}$ Nancy D. Bridges, MD, ${ }^{\mathrm{a}}$ Stephen M. Paridon, MD, ${ }^{\mathrm{a}}$ and \\ Thomas L. Spray, MD, ${ }^{\mathrm{b}}$ Philadelphia, $\mathrm{Pa}$
}

Airway complications after heart-lung transplantation include poor tracheal healing, mucosal slough, stricture, partial disruption, and complete dehiscence of the anastomosis. ${ }^{1-3}$ Complete dehiscence or disruption of the tracheal anastomosis is a rare but often lethal complication. ${ }^{2} \mathrm{We}$ describe the treatment of a patient with acute graft failure and complete tracheal anastomotic disruption after heartlung transplantation.

Clinical summary. A 38-year-old woman had pulmonary hypertension after a ventricular septal defect closure at 5 years of age. She was hospitalized for 3 months before transplantation with severe right-sided heart failure with ascites, hepatomegaly, weight loss, and marked muscle wasting, despite therapy with epoprostenol (Flotan) and inotropic medications. Orthotopic heart-lung transplantation was performed with a graft ischemic time of 305 minutes. The donor trachea was divided 2 rings above the carina, and the recipient's trachea was divided at the level of the carina. An end-to-end tracheal anastomosis was performed with a continuous Prolene suture (Ethicon, Inc, Somerville, NJ) for the membranous portion and interrupted Prolene sutures for the anterior cartilaginous portion. The donor-recipient size match was excellent, and no air leak was noted intraoperatively. Tissue was tacked around the tracheal suture line to separate the anastomosis from the aorta.

Postoperatively, she was maintained on triple immunosuppression therapy consisting of intravenous cyclosporine (Sandimmune; INN: ciclosporin), azathioprine, and steroids; once she was able to tolerate enteral medications, cyclosporine was changed to tacrolimus. She was extubated on postoperative day 4 but required reintubation within 30 hours because of muscle weakness and an inability to phonate, cough, and mobilize respiratory secretions. She was examined with a flexible bronchoscope the next day, revealing an intact, widely patent tracheal anastomosis. There was minimal mucosal necrosis of the membranous portion of the donor trachea. The remainder of the mucosa appeared viable

From the Divisions of Cardiology and Cardiothoracic Surgery, ${ }^{\mathrm{b}}$ The Children's Hospital of Philadelphia, The University of Pennsylvania School of Medicine, Philadelphia, Pa.

Supported in part by a grant from the Daniel M. Tabas Endowed Chair in Cardiothoracic Surgery.

J Thorac Cardiovasc Surg 2001;121:587-8

Copyright (C) 2001 by The American Association for Thoracic Surgery

0022-5223/2001 $\$ 35.00+0 \quad \mathbf{1 2 / 5 4 / 1 1 0 6 8 2}$

doi:10.1067/mtc.2001.110682 and well perfused. Graft function began to deteriorate, with worsening infiltrates on chest radiography and increasing mechanical ventilatory requirements with peak inspiratory pressures as high as $53 \mathrm{~cm} \mathrm{H}_{2} \mathrm{O}$. An open lung biopsy performed 2 weeks after initial transplantation revealed acute cellular rejection, active bronchiolitis obliterans, and severe alveolar damage. She was treated with pulsed steroids and relisted for heart-lung transplantation. In response to progressive graft dysfunction, the immunosuppression therapy was augmented with antithymocyte globulin. During an episode of severe hypoxemia with an inability to ventilate, bronchoscopy revealed a contained tracheal disruption with pulsatile granulation tissue. Thick secretions and thrombus were removed with improved ventilation. Review of the chest radiographs revealed a paratracheal lucency suggestive of anastomotic disruption. A recurrent episode of hypoxemia with difficult ventilation occurred, and repeat bronchoscopy revealed complete disruption of the trachea with a large pulsatile mass posteriorly, which virtually occluded the airway. She was then taken to the operating room for further exploration.

The exploratory operation was done through a median sternotomy with the aid of cardiopulmonary bypass. A large mediastinal hematoma was evacuated, and complete dehiscence of the tracheal anastomosis, with separation of nearly 3 $\mathrm{cm}$ between the ends of the proximal native and distal donor trachea, was found. The distal trachea was destroyed at the level of the bifurcation, with very little cartilage remaining at the carina. The proximal trachea was mobilized extensively to the neck, with care taken to preserve the lateral blood supply. It was not possible to mobilize the distal tracheal because of the inflammatory reaction and scarring. Even with flexion of the neck, it was not possible to approximate the ends of the trachea. Therefore, direct tracheal reconstruction was abandoned, and a 22-mm aortic homograft was used as an interposition graft to bridge the gap between the 2 ends of the trachea. The homograft-tracheal anastomoses were performed with continuous Prolene sutures. The airways were evacuated of a large amount of blood, and ventilation was reinitiated. An airtight anastomosis was ensured, and the lungs were ventilated adequately. She continued to require very high inflating pressures caused by the graft dysfunction and had superior vena cava syndrome caused by a mediastinal clot in conjunction with the high ventilatory pressure.

Three days after placement of the aortic homograft, a suitable heart-lung graft became available, and she underwent repeat transplantation with tracheal reconstruction. Inspection of the aortic homograft revealed the anastomosis to be intact, without air leak, and with mild discoloration from mediastinal 
clot. A bronchoscopic examination performed 5 days after retransplantation revealed a well-perfused distal donor trachea and minimal inflammation. The anastomosis was intact and widely patent, with a very small amount of mucosal sloughing posteriorly on the membranous trachea. The second heart-lung transplant was complicated by hyperammonemia with resultant encephalopathy and electrolyte abnormalities. This was managed successfully with a combination of sodium benzoate and phenylacetate and arginine hydrochloride, as well as continuous intracranial pressure monitoring and venovenous hemodialysis, as has been previously described. ${ }^{4}$ Because of the inability to clear secretions and documented vocal cord paresis, a tracheostomy was performed. She was discharged from the hospital approximately 2 months after the second transplant operation after extensive physical, respiratory, and occupational rehabilitation. The trachea has since been decannulated. At last follow-up and correspondence (1 year after transplantation), she has good graft function, has normal exercise tolerance, and is able to independently perform activities of daily living. There are no neurologic sequelae of her hyperammonemic coma.

Discussion. Healing of the tracheal anastomosis after heart-lung transplantation is dependent on adequate blood supply to the donor trachea. The normal tracheal blood supply is disrupted during harvest of the heart-lung block. After transplantation, blood supply to the donor trachea derives from collateral vessels to the transplanted organ, including coronary-bronchial vessels. A variety of factors may jeopardize this tenuous blood supply, including excess length of the donor trachea and hemodynamic instability. In addition, graft dysfunction with poor lung compliance necessitating the use of high mean airway pressures may impair mucosal perfusion and contribute to poor healing of the tracheal anastomosis. Many operative techniques have been designed to protect the anastomosis. These include omentopexy to neovascularize the bronchial anastomoses and a pericardial flap-plasty or a pericardial fat pad, both of which are used as a protective wrap. ${ }^{2,3}$ Additionally, revascularization with an internal thoracic artery-bronchial artery graft has been described. ${ }^{5}$

Complications, such as partial disruption and anastomotic strictures, can often be managed conservatively, whereas complete tracheal disruption is a catastrophic event presenting with airway obstruction, massive air leaks, or development of aorta-tracheal fistulas with exsanguination. Restoration of airway continuity is the goal of therapy. An end-to-end repair is usually not possible because of necrosis of a significant portion of the donor trachea; this may also preclude mediastinal tracheostomy. The results of prosthetic replacement of the trachea with glass, metal, or silicone rubber have been poor. Placement of tracheal homograft is an option, but availability is limited.

Our patient had been relisted for transplantation because of graft dysfunction, and thus a temporary means to restore airway continuity was needed to bridge to retransplantation. An aortic homograft was placed as an interposition graft to restore airway continuity. The patient underwent successful repeat heart-lung transplant 3 days later.

Aortic homografts are widely available in a variety of sizes. Insertion as an interposition graft in this case was technically straightforward. The homograft tissue is not rigid, and thus continued positive pressure ventilation is necessary. Although the homograft cannot serve as a permanent tracheal replacement, use of an aortic homograft to restore airway continuity is an option for interim management of airway disruption after heart-lung transplantation.

Received for publication July 26, 2000; accepted for publication Aug 1, 2000.

Address for reprints: Thomas L. Spray, MD, Professor and Chief, Division of Cardiothoracic Surgery, The Children's Hospital of Philadelphia, 34th and Civic Center Blvd, Philadelphia, PA 19104.

\section{REFERENCES}

1. Colquhoun IW, Gascoigne AD, Au J, Corris PA, Hilton CJ, Dark JH. Airway complications after pulmonary transplantation. Ann Thorac Surg 1994;57:141-5.

2. Emery RW, Arom KV, von Rueden T, Copeland JG. Use of the pericardial fat pad in pulmonary transplantation. J Card Surg 1990;5:145-8

3. Shumway SJ, Hertz MI, Maynard R, Kshettry VR, Bolman RM. Airway complications after lung and heart-lung transplantation. Transplant Proc 1993;25:1165-6.

4. Berry GT, Bridges ND, Nathanson KL, Kaplan P, Clancy RR, Lichtenstein GR, et al. Successful use of alternate waste nitrogen agents and hemodialysis in a patient with hyperammonemic coma after heart-lung transplantation. Arch Neurol 1999;56:481-4.

5. Yacoub M, Al-Kattan KM, Tadjkarimi S, Eren T, Khaghani A. Medium term results of direct bronchial arterial revascularization using IMA for single lung transplantation (SLT with direct revascularization). Eur J Cardiothoracic Surg 1997;11:1030-6. 